\title{
Quantum Chemical Studies on the Stability of Tetramethyl Ammonium Tetraborohydride
}

Daniel F. Calef

May 1995

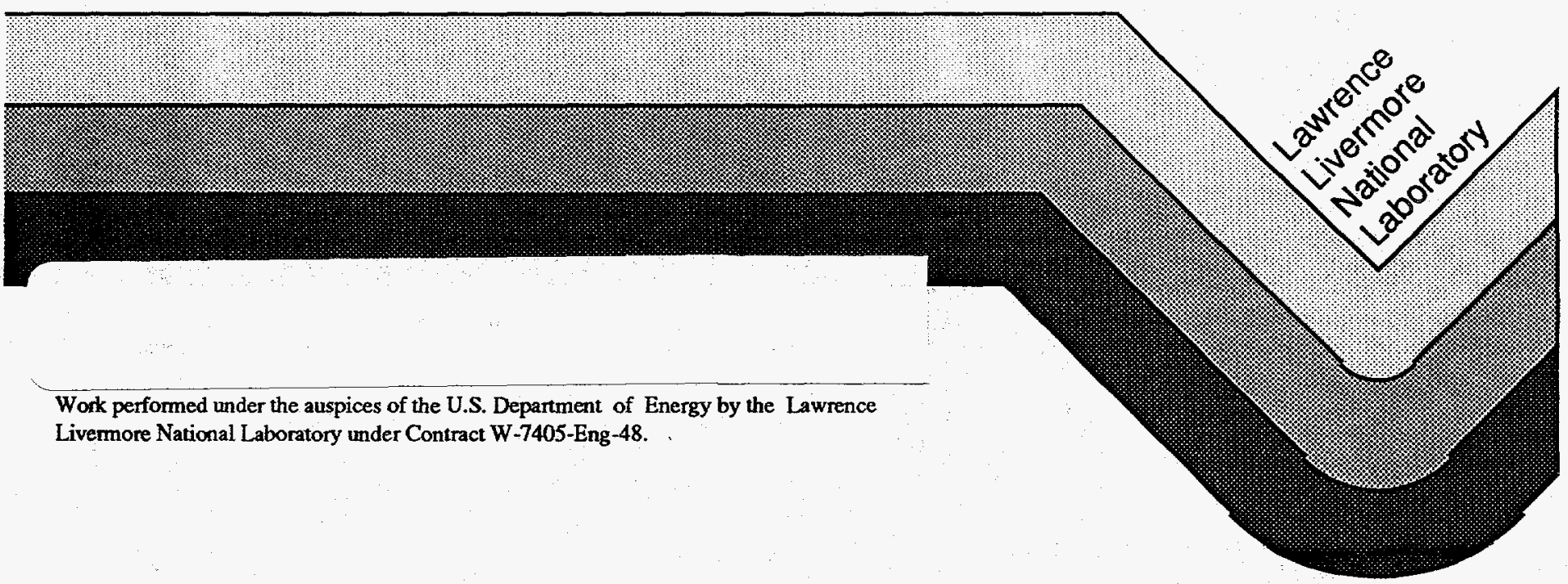




\section{DISCLAIMER}

This report was prepared as an account of work sponsored by an agency of the United States Government. Neither the United States Government nor any agency thereof, nor any of their employees, make any warranty, express or implied, or assumes any legal liability or responsibility for the accuracy, completeness, or usefulness of any information, apparatus, product, or process disclosed, or represents that its use would not infringe privately owned rights. Reference herein to any specific commercial product, process, or service by trade name, trademark, manufacturer, or otherwise does not necessarily constitute or imply its endorsement, recommendation, or favoring by the United States Government or any agency thereof. The views and opinions of authors expressed herein do not necessarily state or reflect those of the United States Government or any agency thereof. 


\section{DISCLAIMER}

Portions of this document may be illegible in electronic image products. Images are produced from the best available original document. 


\title{
Quantum Chemical Studies on the Stability of Tetramethyl Ammonium Tetraborohydride
}

\author{
Daniel F. Calef \\ Chemistry and Materials Science Directorate \\ Lawrence Livermore Nationai Laboratory \\ University of California \\ P.O. Box 808, Livermore CA 94551
}

\begin{abstract}
Quantum chemical calculations were performed on tetramethyl ammonium tetraborohydride. The material is of interest chemically as is exists as a molecular ionic solid. Calculations show that there exist low energy barrier pathways to decomposition and, hence, that the stability of the material is largely due to crystal forces. Possible sources of this stability are discussed.
\end{abstract}

\section{Introduction}

Tetramethyl ammonium tetraborohydride (TMAB) is a molecular ionic solid. In the solid state it exists as $\left(\mathrm{CH}_{3}\right)_{4} \mathrm{~N}^{+} \cdot \mathrm{BH}_{4}{ }^{-}$. Experimentally it appears to be a relatively stable material, which is somewhat surprising as both components are reactive chemical species. For this purpose a quantum chemical investigation of possible reaction, or decomposition routes was performed. 


\section{Model and Methods}

The quantum chemical calculations discussed in this paper were performed using the General Atomic and Molecular Electronic Structure System (GAMESS) program ${ }^{1}$. This program was ported to the Meiko CS2 parallel computer. The GAMESS code will perform most of the same functions as the more popular Gaussian 92 program $^{2}$, but GAMESS was used as it had been ported to a series of other "massively" parallel computers and it was relatively straightforward to modify it to run on the Meiko CS2. Generally, either 64 or 96 nodes were used.

All calculations discussed were performed using a G6-31** basis set. This basis set is the one normally used for high quality calculations on organic compounds. This generates a basis set consisting of 170 basis functions. In order to gain any speed advantage from the parallel parts of the program, direct Self Consistent Field (SCF) has to be performed. A typical SCF calculation required 7 minutes running of 64 processors and a Muller Plesset (MP2) calculation required 28 minutes. In contrast, the same calculations performed on a Silicon Graphics Inc. Indigo2 workstation, using conventional disk storage of integrals, requires 25 minutes for an SCF and 145 minutes for the MP2 calculation.

\section{Results}

Although TMAB seems to be a relatively small molecule, it still has 22 atoms and would require a computationally impossible task to completely map out the potential energy surface. It is necessary, as well as desirable, to use chemical intuition to guide the search for possible reaction paths. $\mathrm{As}^{\mathrm{BH}_{4}}{ }^{-}$is a common hydride donor, the reaction chosen was

$$
\left(\mathrm{CH}_{3}\right)_{4} \mathrm{~N}^{+} \cdot \mathrm{BH}_{4}^{-} \quad \rightarrow \quad\left(\mathrm{CH}_{3}\right)_{3} \mathrm{~N}+\mathrm{CH}_{4}+\mathrm{BH}_{3}
$$

where three well known and more stable compounds are the products. This would most likely occur via hydride "attack" or transfer from $\mathrm{BH}_{4}{ }^{-}$to one of the methyl groups.

In order to assess the barrier to this reaction, it is necessary to find the transition state. This is not straightforward with a 22 atom system. As there is no direct chemical bonding between the two ionic fragments, one variable that can be used is the relative orientation of the two ions. The calculations presented here envision the reaction occurring by the hydride moving to one of the carbons while the carbon undergoes an "umbrella" inversion to create a methane. For this reason, the system studied was a "linear" reaction complex, where B H C N are constrained to be in a line.

The calculations proceeded by performing independent geometry optimizations of the two ions. The two fragments were then placed in the linear configuration and a saddle point 
search was performed. This proceeds by following a selected eigenvector of the force constant matrix. The structure is optimized in all other "directions." The calculation proceeds until a critical point (all first derivatives zero) has been found where the second derivative matrix as one negative eigenvalue and the rest positive. In general, if no constraints were placed on the geometry, it is very unlikely that the automated procedure in GAMESS would actually find a barrier for a system of 22 atoms. Unless a very spectacular initial guess was made as to which of the 60 odd eigenvectors of the starting configuration was likely to evolve to the transition state, the program will usually get "lost."

After a transition state was found, the eigenvalue corresponding to the "path" down from the saddle can be followed to the reactants and the products. The calculation proceeds until energy minima are found.

All of these calculations were performed at the Hartree Fock level of calculation. At this level, the GAMESS code has analytic forms for the required second derative matrix (generally called a Hessian).

This procedure yielded a set of 29 configurations along the reaction path. In order to find out the importance of post Hartree Fock (or "correlation" effects) a series of MP2 perturbation theory calculations were performed at each of these geometries. The structures were not reoptimized at the MP2 level. These would be called MP2/G6-31** calculations in the quantum chemical literature.

\section{Conclusions}

The results are shown in figure 1. The Hartree Fock and MP2 energies are shown for the 29 points on the reaction points as well as the geometry of the reactants, transition state, and products. The results of the calculation show that for this relative orientation of the two fragments there is a calculated barrier of $11 \mathrm{kcals}$ at the Hartree Fock level and $6 \mathrm{kcals}$ at the MP2 level. It is unlikely that improved post Hartree Fock calculations (i.e. configuration interaction) would change this barrier significantly.

This result should be compared with typical reaction barriers of 50 kcals. As the activation barrier occurs in most expressions for the reaction rate as $\exp (-\mathrm{E} / \mathrm{k} \mathrm{T})$, a small activation energy usually implies a rapid reaction.

If this were the entire barrier to decomposition, TMAB would not be regarded as chemically stable. The observed stability of the material must be arising from an effective barrier that discourages the formation of this complex.

A clue to the origin of the barrier can be found from NMR experiments that have been performed on the related compound $\mathrm{BF}_{4}\left(\mathrm{CH}_{3}\right)_{4} \mathrm{~N}$ in the crystalline state ${ }^{3}$. It is observed that, as expected, the molecule does in fact behave like two separate molecular ions. The motion of the 
two fragments is completely different. The $\mathrm{BF}_{4}$ - entity essentially tumbles or spins freely, even down to very low temperatures. The tetramethyl ammonium ion is essentially locked into an orientation by the crystal forces, and only rarely undergoes a reorientation. This reorientation turns out to be an activated process with a barrier of $6 \mathrm{kcals}$.

This data then suggests that the observed stability of the TMAB is due to a combination of factors: (1) the relatively small barrier to breaking and making chemical bonds calculated here, (2) an electrostatic term from the surrounding ionic lattice (which will tend to stabilize the charged reactants, and hence probably is largest source of stabilization) (3) a barrier to reorientation of ammonium entity, (4) a probabilistic factor expressing how likely the ammonium molecule passes through this orientation during such a flip. The important conclusion is that while the bond breaking and making reaction is only sensitive to the temperature, the other steps are quite environmentally sensitive. For example, these processes will be affected by defects and impurities (such as the proposed reaction products).

The crystal forces and the relative motion of the ions in the solid state can be studied using molecular dynamics and these calculations are underway.

\section{References}

${ }^{1}$ M. W Schmidt, K. K. Baldridge, J. A. Boatz, S. T. Ebert, M. S. Gordon, J. H. Jensen, S. Koseki, N. Matsunga, K. A. Nguyen, S. J. Su, T. L. Windus, J. Comp. Chem., 14, 1347(1993).

${ }^{2}$ GAUSSIAN92, Revision B, M. J. Frisch, G. W Trucks, M. Head-Gordon, P. M. W. Gill, M. W. Wong, J. B. Foresman, B. G. Johnson, H. B Schlegel, M. A. Robb, E. S. Replogle, R. Gomperts, J. L. Andres, K. Raghavachari, J. S. Binkley, C. Gonzalez, R. L. Maartin, D. J. Fox, D. J. Fox, D. J. Defrees, J. Baker, J. J. P. Stewart, J. A. Pople, Gaussian Inc., Pittsburgh, PA, 1992.

${ }^{3}$ S. Torre and P. Ferloni, Z. Naturforsch. 47a 721 (1992).

\section{Figure Captions}

1. Hartree Fock and MP2 energies for the linear complex along the reaction coordinate. Also shown are the geometries for the reactant, transition state, and product complex. 


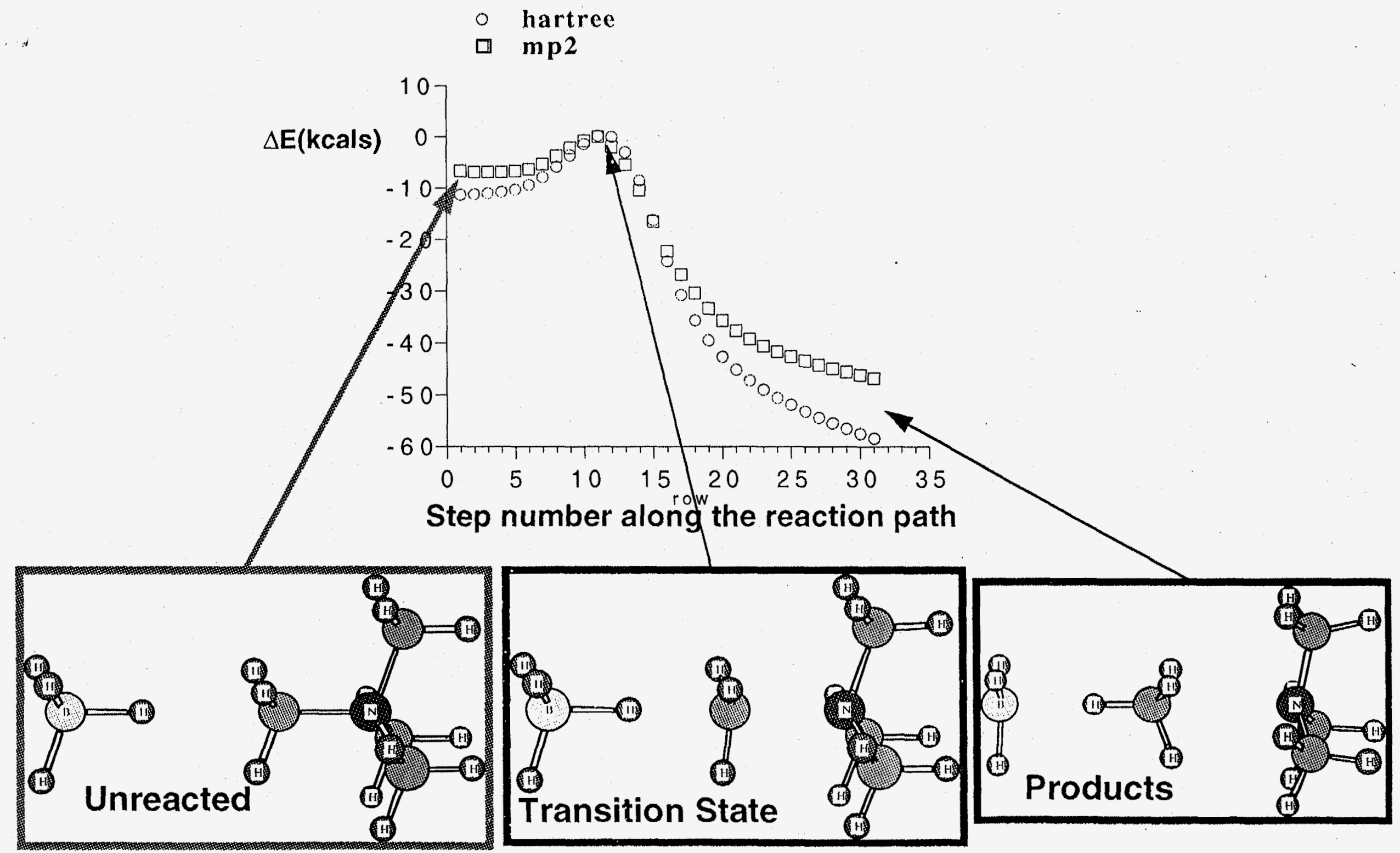

Figure 1. Hartree Fock and MP2 energies for the linear complex along the reaction coordinate. Also shown are the geometries for the reactant, transition state, and product complex. 